\title{
Analysis of recurrence and survival rates in grade 3 endometrioid endometrial carcinoma
}

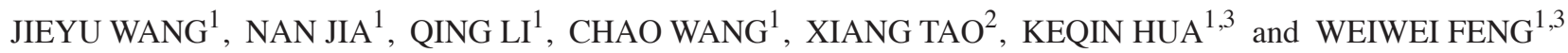 \\ Departments of ${ }^{1}$ Gynecology and ${ }^{2}$ Pathology, Obstetrics and Gynecology Hospital, Fudan University; \\ ${ }^{3}$ Shanghai Key Laboratory of Female Reproductive Endocrine-Related Disease, Fudan University, Shanghai 200011, P.R. China
}

Received March 28, 2015; Accepted April 22, 2016

DOI: $10.3892 / \mathrm{ol} .2016 .4918$

\begin{abstract}
The aim of the present study was to determine risk factors associated with recurrence and survival in patients with grade 3 endometrioid endometrial carcinoma (G3EEC). A retrospective analysis of 117 patients with G3EEC, who were admitted to the Obstetrics and Gynecology Hospital of Fudan University between January 2000 and December 2011, was performed. The $\chi^{2}$ test or Fisher's exact test were used for the comparison of categorical variables. Kaplan-Meier method was used for estimating recurrence-free survival (RFS), disease-specific survival (DSS) and overall survival (OS) rates. Univariate and multivariate Cox proportional hazards model were used to assess the prognostic significance of various patient characteristics. In 117 patients, 16 patients (13.7\%) had tumor recurrence, of which $6(37.5 \%)$ developed local-regional recurrence and $10(62.5 \%)$ developed distant recurrence. Out of the 16 patients with tumor recurrence, 14 (87.5\%) had a recurrence within 3 years of surgery. Statistically significant characteristics affecting RFS, DSS and OS rates were outer half myometrial invasion ( $\mathrm{MI} \geq 50 \%$ ), advanced International Federation of Gynecology and Obstetrics stage, positive lymph node metastasis (PLNM), lymph vascular space invasion, adnexal involvement and characterization as a high-risk group, according to the Gynecologic Oncology Group 99 stratification algorithm. RFS was associated with the depth of cervical mucosa (stromal) involvement. Furthermore, in the
\end{abstract}

Correspondence to: Dr Weiwei Feng, Department of Gynecology, Obstetrics and Gynecology Hospital, Fudan University, 413 Zhaozhou Road, Huangpu, Shanghai 200011, P.R. China

E-mail:wfeng7347@aliyun.com

Abbreviations: G3EEC, grade 3 endometrioid endometrial carcinoma; RFS, recurrence-free survival; DSS, disease-specific survival; OS, overall survival; FIGO, international federation of gynecology and obstetrics; PLNM, positive lymph nodes metastasis; GOG, the gynecologic oncology group; MI, myometrial invasion; LVSI, lymph vascular space invasion; LIR, low-intermediate risk; HIR, high-intermediate risk; HR, high-risk

Key words: endometrioid endometrial cancer, grade 3, myometrial invasion, adnexal involvement, positive lymph nodes metastasis, recurrence, survival multivariate Cox proportional hazards model, significant independent adverse factors for RFS and DSS included MI $\geq 50 \%$ and adnexal involvement. For OS, there were no statistically significant prognostic factors. In conclusion, $\mathrm{MI} \geq 50 \%$ and adnexal involvement are independent prognostic factors for RFS and DSS in G3EEC patients.

\section{Introduction}

Worldwide, 320,000 women are diagnosed with endometrial cancer each year and there are 76,000 mortalities associated with endometrial cancer, which results in it being the sixth most common cancer in women. Compared with an incidence rate of $0.6 \%$ in developing countries, in developed countries the incidence rate of endometrial cancer is $1.6 \%$ (1). The incidence of endometrial cancer is increasing, which has been hypothesized to be associated with an increased life expectancy and increasing incidence of obesity (2).

Endometrioid endometrial cancer (EEC) accounts for $\sim 80 \%$ of endometrial cancers. EEC tumors are usually estrogen-dependent, often low-grade, diagnosed at an early stage and are associated with an improved prognosis $(3,4)$. However, a previous study has revealed that certain survival outcomes, including recurrence-free survival (RFS) and disease-specific survival (DSS), are similar among malignant mixed mullerian tumors, high-grade EEC, clear cell (CC) and uterine serous carcinoma (USC) subtypes of endometrial cancer (5). Del Carmen et al (6) also reported that patients with grade 3 EEC (G3EEC) have a higher relapse rate compared with patients with low-grade endometrioid adenocarcinoma of the uterus. Therefore, G3EEC is considered as a high-risk endometrial cancer, along with USC and CC; however, factors associated with behavior and recurrence of G3EEC remain unclear.

The Gynecologic Oncology Group (GOG) divided endometrial cancer into three subgroups based on surgical-pathological factors and age in order to evaluate the risk of relapse accurately $(7,8)$. A risk stratification algorithm, published in GOG99 (8), has been increasingly used to guide adjuvant therapy for patients with intermediate and high-risk endometrial adenocarcinoma $(9,10)$. However, it is unknown whether this risk stratification algorithm is suitable for G3EEC, and it is unclear if there is an association between conventional prognostic indicators and prognosis among G3EEC patients. 
The present study reviewed patients with G3EEC in order to clarify the risk factors for relapse and mortality in these patients. The aim of the present study was to assess the association of GOG99 risk stratification and various clinicopathological features documented at the time of diagnosis, including depth of myometrial invasion (MI), lymph vascular space invasion (LVSI), International Federation of Gynecology and Obstetrics (FIGO) stage, depth of cervical mucosa (stromal) involvement (CMI), adnexal involvement, pelvic lymph node metastasis (PLNM) and tumor diameter, with the relapse and survival rates of G3EEC patients, and to determine independent factors affecting the recurrence and survival status of patients with G3EEC.

\section{Materials and methods}

Patients. A retrospective review of 117 patients diagnosed with G3EEC at the Obstetrics and Gynecology Hospital of Fudan University (Shanghai, China) between January 2000 and December 2011 was performed. Information on patient demographics, clinical presentation, pathological characteristics, recurrent and survival outcomes was extracted from patient medical records. The institutional review board of the Obstetrics and Gynecology Hospital of Fudan University approved the study. The inclusion criteria for the present study was patients that had undergone comprehensive surgical staging with G3EEC during final pathology evaluation; all paraffin-embedded pathology specimens underwent a pathology review by experienced gynecological pathologists. The exclusion criteria for the present study included patients that had the following characteristics: Non-endometrioid histology; grade (G) 1 or G2 disease; and presence of synchronous cancers. All of the present patients underwent a total hysterectomy and bilateral salpingo-oophorectomy and/or partial omentectomy with or without pelvic and/or para-aortic lymphadenectomy according to the disease stage of the patient. Post-operative adjuvant treatment was determined based on FIGO stage, patient preference and physician suggestion. Standard chemotherapy consisted of paclitaxel and carboplatin for 4-6 cycles and was used in 51 patients. Pelvic radiotherapy and/or vaginal brachytherapy were administered in 6 patients. In total, 39 patients were treated with standard chemotherapy and pelvic radiotherapy and/or vaginal brachytherapy. The post-surgery treatment of 3 patients was unknown, and the remaining 18 patients underwent observation with no treatment following surgery.

Clinicopathological characteristics. Clinicopathological characteristics included age, menopausal status, FIGO stage, depth of MI, tumor diameter, LVSI, depth of CMI, adnexal involvement, para-aortic lymphadenectomy, PLNM and GOG99 risk stratification. Risk stratification was determined by the standards set forth by the GOG99 protocol (8), and patients were either classified as low-risk, low-intermediate risk (LIR), high-intermediate risk (HIR) or high-risk (HR). Low-risk patients were classified as stage IA, G1 and LVSI negative. LIR patients were classified as stages IA, IB or II that did not otherwise meet low-risk or high-intermediate risk criteria. HIR patients were classified as stages IA, IB and II with the following criteria: G2 or G3; LVSI positive; outer half $\mathrm{MI}(\mathrm{MI} \geq 50 \%)$; $\geq 70$ years of age with one other risk factor, $\geq 50$ years of age with two risk factors or any age with three risk factors. HR patients were classified as stage III or IV. The patients in the present study were G3, and therefore were LIR, HIR or HR. FIGO 2009 staging (11) was used, since staging changes were developed in 2014. Recurrent disease was categorized as regional recurrence (intra-pelvic cavity or vagina) and distal recurrence (extra-pelvic only or intra- and extra pelvic). Para-aortic lymph node recurrence was regarded as an extra-pelvic lesion.

Follow-up. Follow-up evaluations, including the date of last follow up, recurrence and the cause of patient mortalities, were collected from the medical records of the patients. All patients were observed until November 2014 or the date that the patient succumbed to a disease. The status of the patients at the end of the follow-up period was defined as no evidence of disease, alive with disease or succumbed to disease. Patients that succumbed to disease were divided into G3EEC-associated mortality, other disease-associated mortality and succumbed while lost to follow-up. The overall survival (OS) rate was defined as the time between surgery and the date of mortality or last follow-up recorded. RFS was defined as the time between surgery and physical or radiographical evidence of disease recurrence. DSS was defined as the time between diagnosis or treatment and G3EEC-associated mortality; patients that succumbed to other causes were not included.

Statistical analysis. The $\chi^{2}$ test and Fisher's exact test were used to analyze categorical variables. Univariate and multivariate Cox proportional hazard regression model were used to assess the effect of the various clinicopathological characteristics on OS, RFS and DSS. Kaplan-Meier method was used to compare categorical predictors within sub-groups of patients. SPSS version 13.0 software (SPSS, Inc., Chicago, IL, USA) was used for statistical analysis. All P-values were two-sided and $\mathrm{P}<0.05$ was considered to indicate a statistically significant difference.

\section{Results}

Clinicopathological characteristics of patients. The clinicopathological characteristics of the 117 patients are listed in Table I. The mean age was 55.6 years (range, $21-80$ years) and the mean follow-up time for all patients was 63 months (range, 2-153 months). According to the 2009 FIGO staging criteria, 84 patients $(71.8 \%)$ were classified as stage I, $12(10.3 \%)$ as stage II, $18(15.4 \%)$ as stage III and $3(2.6 \%)$ as stage IV. In total, 51 patients (43.6\%) had MI $\geq 50 \%$, and LVSI was observed in $35.9 \%$ of patients. Out of the 117 patients, $53(45.3 \%)$ were classified as LIR, $43(36.8 \%)$ as HIR and the remaining 21 patients $(17.9 \%)$ as HR. In total, 72 of the patients $(61.5 \%)$ were menopausal. There was no difference identified in age between recurrent and not-recurrent patients (57.5 vs. 55.3 years; $\mathrm{P}=0.384$ ).

Association between recurrence and clinicopathological characteristics. The overall relapse rate of the present patients with G3EEC was $13.7 \%(16 / 117)$. Of the 16 patients that relapsed, $14(87.5 \%)$ developed recurrence within 3 years of primary treatment. Of all recurrence cases, there were 
Table I. Clinicopathological characteristics of 117 patients with grade 3 endometrioid endometrial cancer.

\begin{tabular}{|c|c|c|c|c|c|}
\hline \multirow[b]{2}{*}{ Characteristic } & \multirow[b]{2}{*}{$\mathrm{n}(\%)$} & \multicolumn{2}{|c|}{ Recurrence } & \multicolumn{2}{|c|}{ Mortality } \\
\hline & & $\mathrm{n}$ & P-value & $\mathrm{n}$ & $\mathrm{P}$-value \\
\hline Age, years & & & 0.788 & & 0.866 \\
\hline$<50$ & $29(24.8)$ & 3 & & 4 & \\
\hline $50-70$ & $78(66.7)$ & 12 & & 8 & \\
\hline$>70$ & $10(8.5)$ & 1 & & 1 & \\
\hline Menopausal status & & & 0.277 & & 1.000 \\
\hline Pre-menopausal & $45(38.5)$ & 4 & & 5 & \\
\hline Post-menopausal & $72(61.5)$ & 12 & & 8 & \\
\hline FIGO stage & & & $0.002^{\mathrm{a}}$ & & $<0.001^{\mathrm{a}}$ \\
\hline I & $84(71.8)$ & 6 & & 4 & \\
\hline II & $12(10.3)$ & 4 & & 1 & \\
\hline III & $18(15.4)$ & 4 & & 6 & \\
\hline IV & $3(2.6)$ & 2 & & 2 & \\
\hline Depth of myometrial invasion & & & $0.005^{\mathrm{a}}$ & & $0.006^{\mathrm{a}}$ \\
\hline Negative & $9(7.7)$ & 1 & & 1 & \\
\hline$<50 \%$ & $57(48.7)$ & 2 & & 1 & \\
\hline$\geq 50 \%$ & $51(43.6)$ & 13 & & 11 & \\
\hline Tumor diameter, $\mathrm{cm}$ & & & 0.553 & & 0.511 \\
\hline$\leq 2$ & $32(27.4)$ & 3 & & 2 & \\
\hline$>2$ & $83(70.9)$ & 12 & & 11 & \\
\hline Unknown & $2(1.7)$ & 1 & & 0 & \\
\hline Cervical involvement & & & 0.066 & & 0.408 \\
\hline Negative & $88(75.2)$ & 9 & & 8 & \\
\hline Mucosal & $9(7.7)$ & 2 & & 2 & \\
\hline Stromal & $20(17.1)$ & 5 & & 3 & \\
\hline LVSI & & & 0.092 & & $0.013^{\mathrm{a}}$ \\
\hline Negative & $75(64.1)$ & 7 & & 4 & \\
\hline Positive & $42(35.9)$ & 9 & & 9 & \\
\hline PLNM & & & 0.056 & & $<0.001^{\mathrm{a}}$ \\
\hline Negative & $93(79.5)$ & 11 & & 6 & \\
\hline Positive & $17(14.5)$ & 5 & & 7 & \\
\hline Unknown & $7(6.0)$ & 0 & & 0 & \\
\hline Adnexal involvement & & & 0.043 & & $0.020^{\mathrm{a}}$ \\
\hline Negative & $106(90.6)$ & 12 & & 4 & \\
\hline Positive & $11(9.4)$ & 4 & & 9 & \\
\hline Risk stratification & & & $0.010^{\mathrm{a}}$ & & $<0.001^{\mathrm{a}}$ \\
\hline LIR & $53(45.3)$ & 2 & & 1 & \\
\hline HIR & $43(36.8)$ & 8 & & 4 & \\
\hline HR & $21(17.9)$ & 6 & & 8 & \\
\hline Para-aortic lymphadenectomy & & & 1.000 & & 0.356 \\
\hline Negative & $104(88.9)$ & 14 & & 13 & \\
\hline Positive & $13(11.1)$ & 2 & & 0 & \\
\hline Adjuvant therapy & & & 0.712 & & 0.868 \\
\hline Observation & $18(15.4)$ & 1 & & 1 & \\
\hline Chemotherapy & $51(43.6)$ & 7 & & 6 & \\
\hline Radiotherapy & $6(5.1)$ & 1 & & 1 & \\
\hline Chemoradiotherapy & $39(33.3)$ & 7 & & 5 & \\
\hline Unknown & $3(2.6)$ & 0 & & 0 & \\
\hline
\end{tabular}

FIGO, International Federation of Gynecology and Obstetrics; LVSI, lymph vascular space invasion; PLNM, pelvic lymph node metastasis; LIR, low-intermediate risk; HIR, high-intermediate risk; HR, high-risk. ${ }^{\mathrm{a}} \mathrm{P}<0.05$. 
Table II. Univariate Cox proportional hazards analysis for the risk of tumor recurrence, disease-specific survival and overall survival rates associated with various clinicopathological characteristics.

\begin{tabular}{|c|c|c|c|c|c|c|}
\hline \multirow[b]{2}{*}{ Characteristic } & \multicolumn{2}{|c|}{ Recurrence-free survival } & \multicolumn{2}{|c|}{ Disease-specific survival } & \multicolumn{2}{|c|}{ Overall survival } \\
\hline & HZR (95\% CI) & P-value & HZR (95\% CI) & P-value & HZR $(95 \%$ CI) & P-value \\
\hline \multicolumn{7}{|l|}{ Age, years } \\
\hline$\leq 60$ vs. $>60$ & $1.57(0.56-4.41)$ & 0.394 & $1.54(0.57-4.18)$ & 0.396 & $1.74(0.62-4.84)$ & 0.291 \\
\hline \multicolumn{7}{|l|}{ Menopausal status } \\
\hline Pre vs. post & $1.82(0.58-5.72)$ & 0.305 & $1.87(0.60-5.82)$ & 0.277 & $1.25(0.43-3.66)$ & 0.686 \\
\hline \multicolumn{7}{|l|}{ Depth of MI } \\
\hline Negative/ $<50 \%$ vs. $\geq 50 \%$ & $6.59(1.88-23.15)$ & $0.003^{\mathrm{a}}$ & $8.06(1.79-36.43)$ & $0.007^{\mathrm{a}}$ & $6.70(1.92-23.33)$ & $0.003^{\mathrm{a}}$ \\
\hline \multicolumn{7}{|l|}{ LVSI } \\
\hline Negative vs. positive & $2.70(1.01-7.25)$ & $0.049^{\mathrm{a}}$ & $4.48(1.38-14.55)$ & $0.013^{\mathrm{a}}$ & $3.60(1.33-9.73)$ & $0.012^{\mathrm{a}}$ \\
\hline \multicolumn{7}{|l|}{ Adnexal involvement } \\
\hline Negative vs. positive & $4.74(1.50-14.92)$ & $0.008^{\mathrm{a}}$ & $6.04(1.81-20.14)$ & $0.003^{\mathrm{a}}$ & $4.30(1.36-13.57)$ & $0.013^{\mathrm{a}}$ \\
\hline \multicolumn{7}{|l|}{ Cervical involvement } \\
\hline Negative/mucosal vs. interstitial & $3.25(1.18-8.95)$ & $0.023^{\mathrm{a}}$ & $1.64(0.45-5.96)$ & 0.456 & $1.70(0.55-5.24)$ & 0.353 \\
\hline \multicolumn{7}{|l|}{ PLNM } \\
\hline Negative vs. positive & $3.90(1.34-11.36)$ & $0.013^{\mathrm{a}}$ & $9.67(3.21-29.11)$ & $<0.001^{\mathrm{a}}$ & $5.57(2.10-14.77)$ & $0.001^{\mathrm{a}}$ \\
\hline \multicolumn{7}{|l|}{ Tumor diameter, $\mathrm{cm}$} \\
\hline$<2$ vs. $\geq 2$ & $1.69(0.47-6.09)$ & 0.421 & $1.83(0.52-6.51)$ & 0.348 & $1.66(0.46-5.97)$ & 0.437 \\
\hline \multicolumn{7}{|l|}{ Para-aortic lymphadenectomy } \\
\hline Negative vs. positive & $1.39(0.31-6.25)$ & 0.667 & $1.38(0.31-6.20)$ & 0.674 & $0.04(0.00-90.54)$ & 0.417 \\
\hline
\end{tabular}

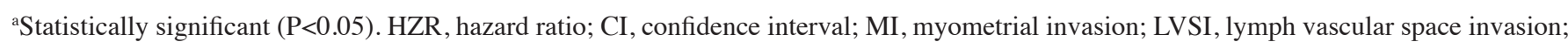
PLNM, pelvic lymph node metastasis.
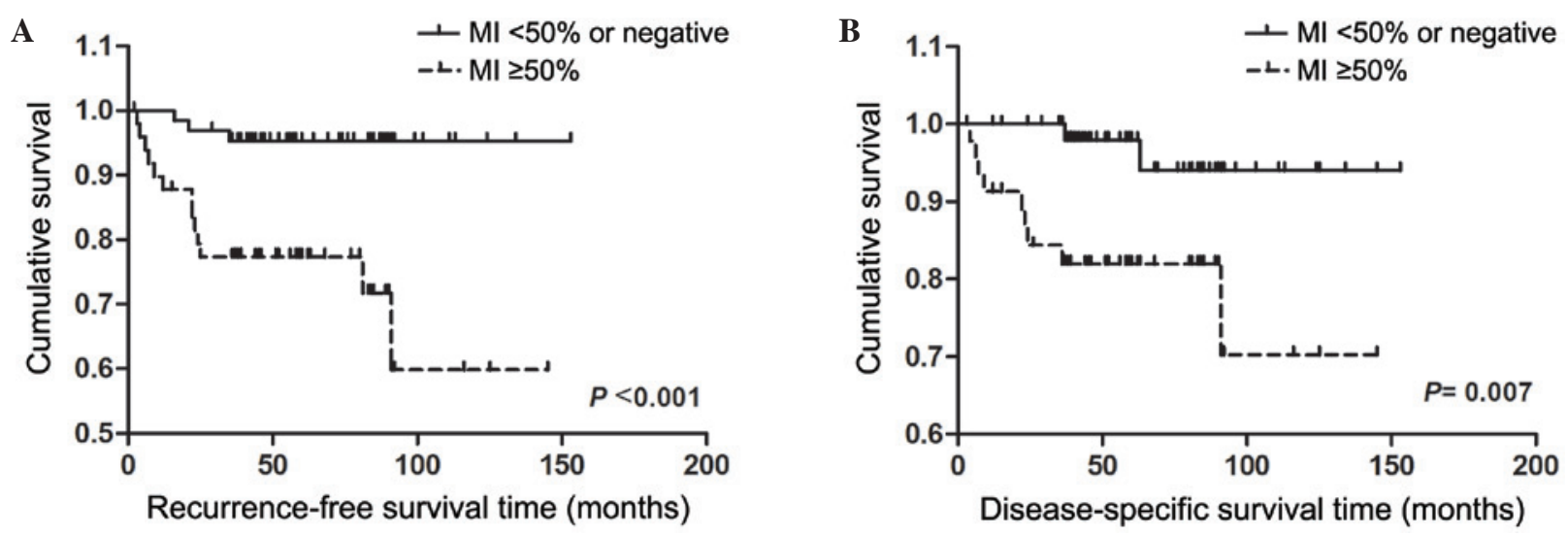

Figure 1. (A) Recurrence-free survival and (B) disease-specific survival rates in patients with grade 3 endometrioid endometrial cancer, according to the depth of MI. MI, myometrial invasion.

$6(37.5 \%)$ regional recurrent patients, including 5 patients in whom recurrence was restricted to the pelvic cavity and 1 patient that had recurrence restricted to the vagina, and $10(55.6 \%)$ distal recurrence patients, including 9 patients with extra-pelvic disease and 1 patient with intra- and extra-pelvic recurrence. Patterns of distal recurrence included lung $(n=4)$, abdomen $(n=1)$, bone $(n=2)$, liver $(n=2)$ and bowel $(n=1)$. The mean time to regional recurrence (intra-pelvic only) and distal recurrence (intra- and extra-pelvic) was not significantly different (12.5 vs. 32.6 months; $\mathrm{P}=0.068)$. Of 16 recurred cases, 10 patients $(62.5 \%)$ succumbed to G3EEC, 5 patients were $(31.3 \%)$ alive with disease and 1 patient succumbed to cerebral hemorrhage. Recurrence and mortality of patients with G3EEC were additionally evaluated for the 117 patients in association with GOG99 risk categories.

Association between risk factors and patient outcome. Table II provides the results of the Cox univariate analysis with corresponding hazard ratio (HZR) and $95 \%$ confidence intervals (CI) for predicting relapse and survival status. MI, FIGO 
Table III. Multivariate Cox proportional hazards analysis for the risk of tumor recurrence, disease-specific survival and overall survival rates associated with various clinicopathological characteristics.

\begin{tabular}{|c|c|c|c|c|c|c|}
\hline \multirow[b]{2}{*}{ Characteristic } & \multicolumn{2}{|c|}{ Recurrence-free survival } & \multicolumn{2}{|c|}{ Disease-specific survival } & \multicolumn{2}{|c|}{ Overall survival } \\
\hline & HZR $(95 \% \mathrm{CI})$ & $\mathrm{P}$-value & HZR $(95 \% \mathrm{CI})$ & P-value & HZR (95\% CI) & P-value \\
\hline \multicolumn{7}{|l|}{ Age, years } \\
\hline$\leq 60$ vs. $>60$ & $0.76(0.19-3.05)$ & 0.699 & $0.72(0.18-2.84)$ & 0.640 & $1.00(0.20-4.85)$ & 0.996 \\
\hline \multicolumn{7}{|l|}{ Menopausal status } \\
\hline Pre vs. post & $2.41(0.59-9.88)$ & 0.220 & $2.93(0.71-12.03)$ & 0.136 & $1.50(0.32-6.94)$ & 0.604 \\
\hline \multicolumn{7}{|l|}{ Depth of MI } \\
\hline Negative/ $<50 \%$ vs. $\geq 50 \%$ & $4.91(1.02-23.57)$ & $0.047^{\mathrm{a}}$ & $4.83(1.06-22.04)$ & $0.042^{\mathrm{a}}$ & $3.87(0.77-19.35)$ & 0.099 \\
\hline \multicolumn{7}{|l|}{ LVSI } \\
\hline Negative vs. positive & $1.92(0.53-6.90)$ & 0.318 & $2.08(0.59-7.33)$ & 0.254 & $2.17(0.61-7.77)$ & 0.234 \\
\hline \multicolumn{7}{|l|}{ Adnexal involvement } \\
\hline Negative vs. positive & $4.29(1.07-17.15)$ & $0.040^{\mathrm{a}}$ & $4.04(1.04-15.60)$ & $0.043^{\mathrm{a}}$ & $2.62(0.61-11.21)$ & 0.196 \\
\hline \multicolumn{7}{|l|}{ Cervical involvement } \\
\hline Negative/mucosal vs. interstitial & $1.15(0.29-4.51)$ & 0.847 & $1.05(0.27-4.05)$ & 0.943 & $0.51(0.09-2.72)$ & 0.428 \\
\hline \multicolumn{7}{|l|}{ PLNM } \\
\hline Negative vs. positive & $1.77(0.42-7.56)$ & 0.440 & $2.89(0.78-10.66)$ & 0.111 & $2.21(0.58-8.41)$ & 0.246 \\
\hline \multicolumn{7}{|l|}{ Tumor diameter, $\mathrm{cm}$} \\
\hline$<2$ vs. $\geq 2$ & $0.45(0.10-2.13)$ & 0.317 & $0.50(0.11-2.26)$ & 0.370 & $0.72(0.17-3.03)$ & 0.653 \\
\hline \multicolumn{7}{|l|}{ Para-aortic lymphadenectomy } \\
\hline Negative vs. positive & $1.18(0.24-5.88)$ & 0.840 & $0.98(0.20-4.87)$ & 0.982 & $0.83(0.27-4.16)$ & 0.983 \\
\hline
\end{tabular}

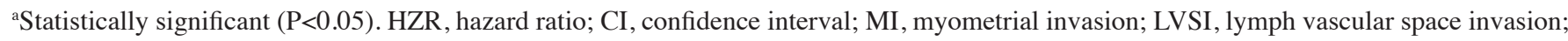
PLNM, pelvic lymph node metastasis.
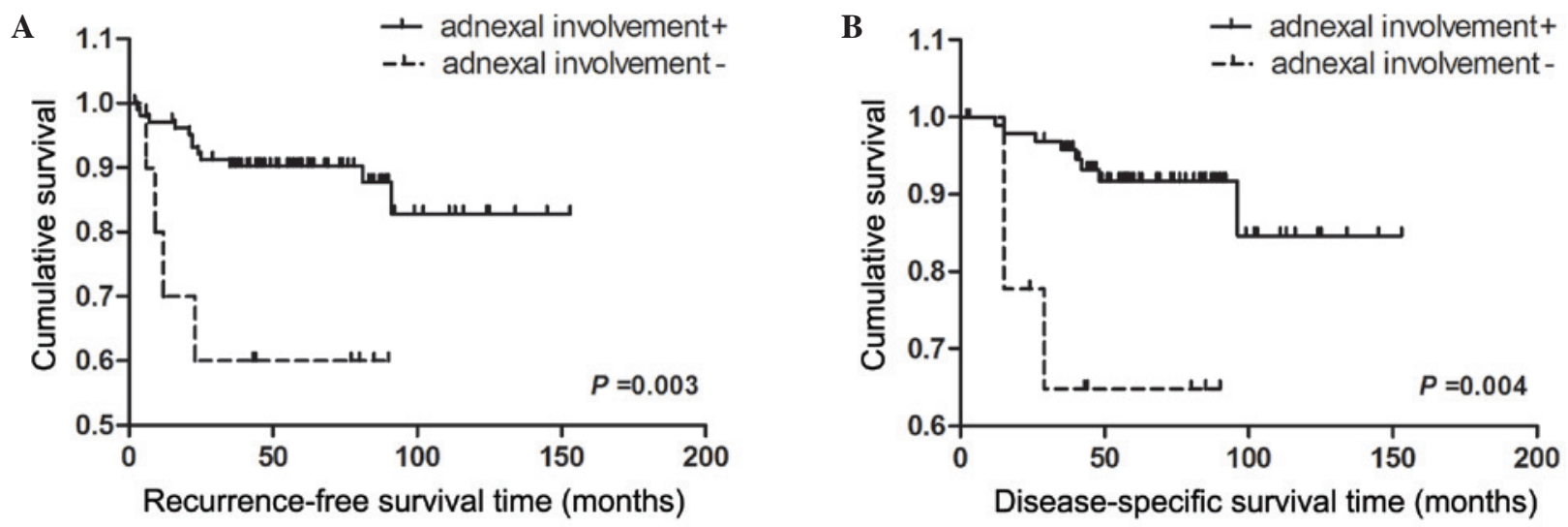

Figure 2. (A) Recurrence-free survival and (B) disease-specific survival rates in patients with grade 3 endometrioid endometrial cancer, according to adnexal involvement.

stages, LVSI, adnexal involvement, PLNM, CMI and risk stratification were significantly associated with an increased risk of recurrence using univariate analysis $(\mathrm{P}<0.05)$. DSS and OS rates were also associated with these characteristics, with the exception of CMI and risk stratification when divided into LIR and HIR groups.

Multivariate analysis (Table III) identified $\mathrm{MI} \geq 50 \%$ (HZR, 4.91; 95\% CI, 1.02-23.57; $\mathrm{P}=0.047)$ and adnexal involvement (HZR, 4.29; 95\% CI, 1.07-17.15; P=0.040) were associated with RFS. For DSS, MI $\geq 50 \%$ (HR, 4.83; 95\% CI, 1.06-22.04;
$\mathrm{P}=0.042)$ and adnexal involvement (HR, 4.04; 95\% CI, 1.04$15.60 ; \mathrm{P}=0.043)$ were also independent prognostic factors.

For all G3EEC patients, the OS, RFS and DSS were 70.8, 80.5 and $81.9 \%$, respectively. When survival curves were stratified by MI, there was a significant increase in RFS and DSS rates for patients with tumors with $\mathrm{MI}<50 \%$ compared with those with $\mathrm{MI} \geq 50 \%$ (Fig. 1). In addition, adnexal involvement was a negative prognostic factor for RFS and DSS (Fig. 2). Furthermore, OS rate, RFS and DSS clearly differed between the LIR, HIR and HR groups, as shown in Fig. 3. 

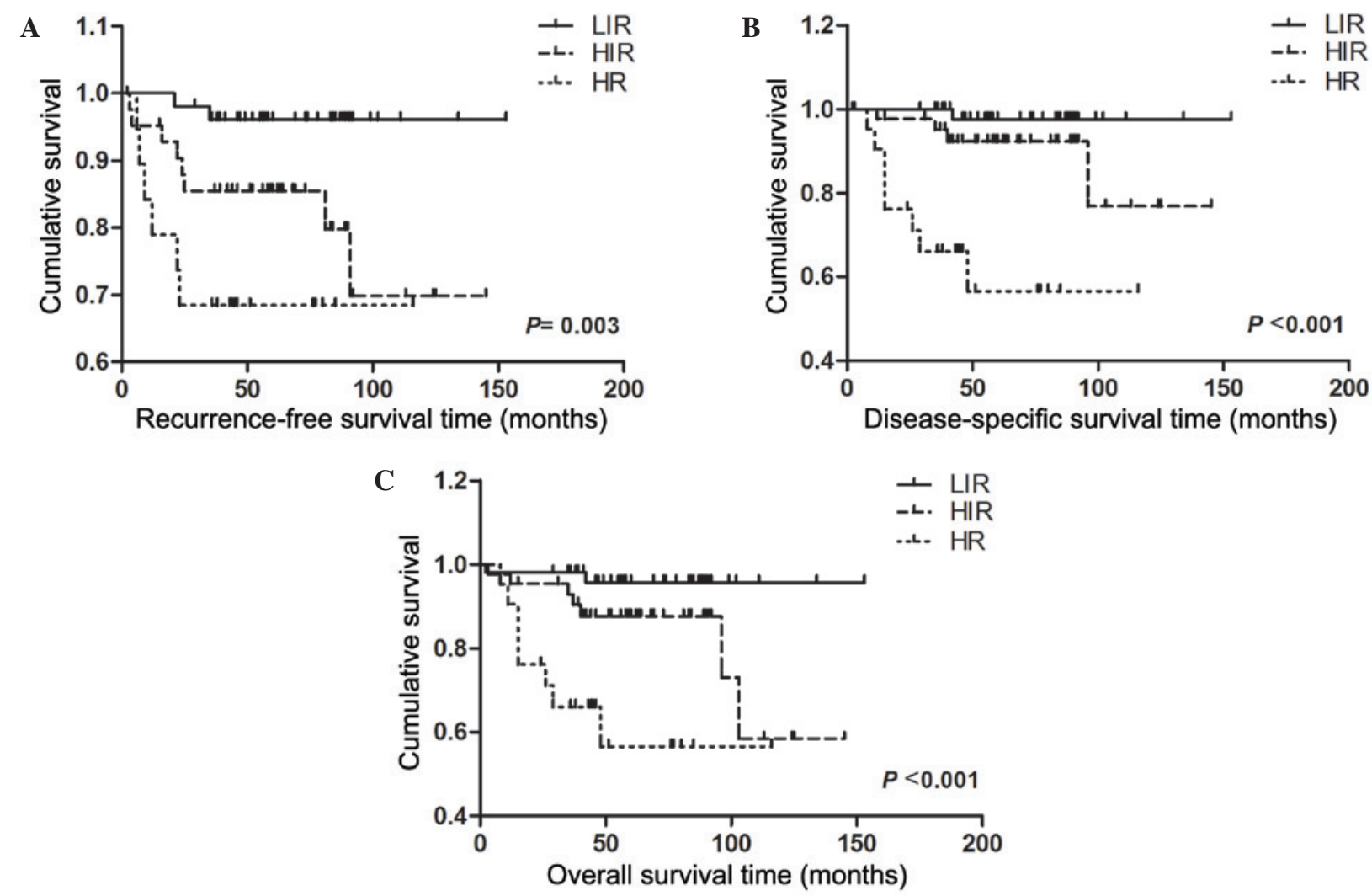

Figure 3. (A) Recurrence-free survival, (B) disease-specific survival and (C) overall survival rates in patients with grade 3 endometrioid endometrial cancer, according to LIR, HIR and HR risk stratification groups. LIR, low-intermediate risk; HIR, high-intermediate risk; HR, high risk.

\section{Discussion}

The present retrospective review evaluated a group of 117 G3EEC patients treated in a single institution, and examined the prognostic variables associated with recurrence and survival status. The present study identified a recurrence rate of $\sim 13.7 \%$, which is lower than the $19.6 \%$ (10/51) reported by a previous study by Kim et al (12). This difference may be due to the FIGO stages identified. In the present study, early stage (I and II) tumors accounted for $82.1 \%$ of the patients, which is increased compared with $80.4 \%$ observed by $\mathrm{Kim}$ et al (12). However, Gayar et al (13) reported a recurrence rate of $23.6 \%$ (26/110) in G3 patients with early-stage tumors (FIGO stage I-II), which is increased compared with $13.7 \%$ (16/117 patients) observed in the present study. The OS rate in the present study was $70.8 \%$, which was lower compared with $81.0 \%$ identified in a previous study including patients of all grades (14). This difference supports the hypothesis that G3 patients have a worse prognosis.

Although the vaginal cuff is the most common site for tumor recurrence following a hysterectomy in early-stage endometrial carcinoma patients, with locoregional recurrence rates of 4-8\% (8,15), Rasool et al (16) reported a high rate of distant recurrence in patients with stage I G3EEC and a risk of extra-pelvic recurrence of $80 \%$, which is higher compared with local regional recurrence. In a previous study of 28 cases with stage IV G3EEC, recurrence was observed in 6 cases, and all of these were distant recurrence (17). With a relatively large sample size, the present study suggests that both regional and distal recurrence occurs in patients with G3EEC. Of the
16 patients in the present study with G3 tumors that had recurrence, 10 patients (55.6\%) had a component of distant failure, which is similar to the results demonstrated by Kim et al (12), who revealed 1 local regional, 3 lymphatic and 6 hematogenous distant failure recurrences in 10 G3EEC patients. Therefore, the majority of G3 patients have a tendency for distant recurrence and may succumb to disease, suggesting that current loco-regional adjuvant treatment strategies, including brachytherapy and pelvic radiotherapy, are not beneficial and additional systemic therapies are required.

In agreement with other studies (18-20), which demonstrated that MI is associated with an increasing risk of tumor failure and has a negative affect on survival, the present study suggests that MI is a clear adverse predictor of tumor recurrence and survival end points. Depth of MI has been recognized as an independent prognostic factor for patients with stage I and II endometrial adenocarcinoma (21) and is the clearest predictor of distant failure and mortality from disease in stage I endometrial cancer (18). MI $\geq 66 \%$ was identified as the only independent predictor of disease-free survival (DFS) and RFS in 229 patients with stage I epithelial endometrial cancer of all subtypes (18). Additionally, in a previous study of 213 patients with endometrial cancer, MI $>50 \%$ is clear risk for extra-uterine metastases (22). Furthermore, patients with MI $>50 \%$ had a $>6$-fold higher prevalence of pelvic lymph node metastases compared with patients with MI <50\% (22). Depth of MI has also been revealed to be an independent predictor of pelvic relapse in a multivariate analysis (23). Hematogenous dissemination is defined as tumor spread to the lung, liver or other site via hematogenous routes. Mariani et al (24) reported 
that the presence of deep MI was the clearest predictor of hematogenous dissemination in corpus cancer. The present study also revealed that MI $\geq 50 \%$ was a statistically significant characteristic associated with a decrease in DSS and RFS rates. In the current study, the RFS and DSS rates were 59.8 and $70.2 \%$ for patients with deep MI ( $\geq 50 \%)$, compared to 95.3 and $94.0 \%$ for patients with superficial MI $(<50 \%)$, respectively. In conclusion, $\mathrm{MI} \geq 50 \%$ is the most important prognostic factor for G3EEC patients, and patients with deep MI require a more aggressive therapy and more frequent observation to monitor the presence or absence of recurrence.

However, there are contrary views concerning the importance of MI. Certain studies have demonstrated that in patients with early stage endometrial cancer, MI does not appear to be associated with patient outcomes, including RFS (25), DSS (25), OS $(25,26)$ and DFS (26). Chattopadhyay et al (20) reported that tumor size was the only independent predictor for distant recurrence and mortality from disease in stage I EEC, while MI only predicted distant failure. In the present study, distant failure was the main G3EEC recurrence site; therefore, the study by Chattopadhyay et al supports the present results to a certain extent. Overall, the inconsistencies observed with MI may be due to differences in stage or grade amongst selected patients and the various definitions of MI; depths of $>1 / 3$ (26), $>50 \%$ (22) and $\geq 66 \%$ (18) are all regarded as cut-off values for deep MI in various studies, indicating that in endometrial cancer, the depth of MI has various roles according to the severity of the disease. In previous studies MI has been associated with certain biochemical indicators, including levels of free insulin-like growth factor-1 plasma (27), nucleoporin (88kDa)-mRNA expression (28) and endoplasmic reticulum- $\beta$ (29). An association was also demonstrated between tumor-associated macrophages and depth of MI (30).

The present study demonstrated that, in G3EEC patients, adnexal involvement is also a risk factor for recurrence and disease-related mortality. Adnexal metastasis is associated with omental metastasis (31) and para-aortic lymph node metastasis (32). The risk of tumor recurrence in patients with adnexal involvement is 4.29-fold higher compared with patients without adnexal involvement, which is consistent with the results demonstrated by Hétu et al (33), who reported that patients with metastasis to the adnexa have a higher risk of recurrence. With regard to DSS, Jobsen et al (34) reported that the 7-year DSS rate was $71.8 \%$ for patients with adnexal involvement in stage IIIA endometrial carcinoma. In the present study, patients with positive adnexal involvement had a 4.04-fold higher risk of disease-related mortality compared with patients without adnexal involvement.

The GOG99 trial was a randomized controlled trial comparing postoperative pelvic radiotherapy to no additional treatment in stage IB, IC or IIA endometrial carcinoma of any histological grade (8). GOG99 revealed that age, grade, LVSI and MI are factors that influence the risk of relapse. In the present study, risk stratification was revealed to have a negative association with the outcome of patients using univariate cox proportional hazards analysis. In a previous study, HIR patients had a decreased OS rate compared with patients that were not classified as HIR (35). The present results revealed that the prognosis of patients clearly differed between the
LIR, HIR and HR groups in G3EEC. DSS, OS rate and RFS were 97.6, 95.7 and $96.1 \%$ for the LIR group, 76.9, 58.4 and $69.8 \%$ in the HIR group, and 56.6, 56.6 and $68.4 \%$ for the HR group, respectively. However, in the univariate cox proportional hazards analysis, compared with the LIR group, patients in the HIR and HR groups demonstrated 5.30 and 11.04-fold decrease in RFS, 5.01 and 27.41-fold decrease in DSS and 4.20 and 13.14-fold decrease in OS rates, respectively. In the multivariate Cox proportional hazards analysis, risk classification was not an independent factor, which may be due to risk stratification being associated with conventional prognostic indicators $(8,36)$. Therefore, the present study hypothesizes that the criteria of risk stratification is associated with the prognostic status of G3EEC patients, but is not an independent factor.

In conclusion, the present results suggest that in patients with G3EEC MI $\geq 50 \%$ and adnexal involvement are significant risk factors affecting RFS and DSS. The present study has also demonstrated that in patients with G3EEC, GOG99 risk stratification had an association with the patient outcome of relapse and survival. Based on these findings, the risk factors of G3EEC evaluated by the present study may be used to evaluate the outcome of patients. Patients with MI $\geq 50 \%$ or positive adnexal involvement should undergo more aggressive therapy and a more rigorous follow-up plan. The limitations of the present study include the inherent biases of a retrospective single institution study design. Furthermore, the study population was relatively small due to the strict selection criteria. Additional larger prospective studies examining the same type of patient population are required to verify the present findings.

\section{Acknowledgement}

The present study was supported by the National Natural Science Foundation of China (Beijing, China; grant no. 30973185).

\section{References}

1. Galaal K, Al Moundhri M, Bryant A, Lopes AD and Lawrie TA: Adjuvant chemotherapy for advanced endometrial cancer. Cochrane Database Syst Rev 5: CD010681, 2014.

2. Emily K, Clark L, Franasiak J, Bae-Jump V and Gehrig P: Obesity portends a significantly higher risk of recurrence in women with grade 3 endometrial carcinoma: A call to action. Gynecolo Oncol 125 (Supp 1): S158, 2012.

3. Sorosky JI: Endometrial cancer. Obstetr Gynecol 120: 383-397, 2012.

4. Tangjitgamol S1, Anderson BO, See HT, Lertbutsayanukul C, Sirisabya N, Manchana T, Ilancheran A, Lee KM, Lim SE, Chia YN, et al; Asian Oncology Summit: Management of endometrial cancer in Asia: consensus statement from the Asian Oncology Summit 2009. Lancet Oncol 10: 1119-1127, 2009.

5. Felix AS, Stone RA, Bowser R, Chivukula M, Edwards RP, Weissfeld JL and Linkov F: Comparison of survival outcomes between patients with malignant mixed mullerian tumors and high-grade endometrioid, clear cell, and papillary serous endometrial cancers. Int J Gynecol Cancer 21: 877-884, 2011.

6. Del Carmen MG, Boruta DM II and Schorge JO: Recurrent endometrial cancer. Clin Obstet Gynecol 54: 266-277, 2011.

7. Morrow CP, Bundy BN, Kurman RJ, Creasman WT, Heller P, Homesley HD and Graham JE: Relationship between surgical-pathological risk factors and outcome in clinical stage I and II carcinoma of the endometrium: A gynecologic oncology group study. Gynecol Oncol 40: 55-65, 1991. 
8. Keys HM, Roberts JA, Brunetto VL, Zaino RJ, Spirtos NM, Bloss JD, Pearlman A, Maiman MA and Bell JG; Gynecologic Oncology Group: A phase III trial of surgery with or without adjunctive external pelvic radiation therapy in intermediate risk endometrial adenocarcinoma: A gynecologic oncology group study. Gynecol Oncol 92: 744-751, 2004.

9. Ioffe Y, Delic L, Amneus M, Leuchter R, Karlan B, Li A, Walsh C, Lentz S, Farias-Eisner R and Cass I: Before and after GOG 99: Did our practice patterns for treatment of intermediate risk endometrial adenocarcinoma change? Gynecol Oncol 116: $595,2010$.

10. Rankins NC, Secord AA, Jewell E, Havrilesky LJ, Soper JT and Myers E: Cost-effectiveness of adjuvant radiotherapy in intermediate risk endometrial cancer. Gynecol Oncol 106 388-393, 2007.

11. Edge S, Byrd DR, Compton CC, Fritz AG, Greene FL and Trotti A (eds): Gynecologic Sites - Corpus Uteri. In: AJCC Cancer Staging Manual. 7th edition. Springer, New York, p403, 2010.

12. Kim HJ, Kim TJ, Lee YY, Choi CH, Lee JW, Bae DS and Kim BG: A comparison of uterine papillary serous, clear cell carcinomas, and grade 3 endometrioid corpus cancers using 2009 FIGO staging system. J Gynecol Oncol 24: 120-127, 2013.

13. Gayar OH, Patel S, Schultz D, Mahan M, Rasool N and Elshaikh MA: The impact of tumor grade on survival end points and patterns of recurrence of 949 patients with early-stage endometrioid carcinoma: A single institution study. Int J Gynecol Cancer 24: 97-101, 2014.

14. Tejerizo-García A, Jiménez-López JS, Muñoz-González JL, Bartolomé-Sotillos S, Marqueta-Marqués L, López-González G and Gómez JF: Overall survival and disease-free survival in endometrial cancer: Prognostic factors in 276 patients. Onco Targets Ther 9: 1305-1313, 2013.

15. Creutzberg CL, van Putten WL, Koper PC, Lybeert ML, Jobsen JJ, Wárlám-Rodenhuis CC, De Winter KA, Lutgens LC, van den Bergh AC, van de Steen-Banasik E, et al: Surgery and postoperative radiotherapy versus surgery alone for patients with stage-1 endometrial carcinoma: Multicentre randomised trial. PORTEC study group. Post operative radiation therapy in endometrial carcinoma. Lancet 355: 1404-1411, 2000.

16. Rasool N, Fader AN, Seamon L, Neubauer NL, Shahin FA, Alexander HA, Moore K, Moxley K, Secord AA, Kunos C, et al: Stage I, grade 3 endometrioid adenocarcinoma of the endometrium: An analysis of clinical outcomes and patterns of recurrence. Gynecol Oncol 116: 10-14, 2010.

17. Long KC, Zhou Q, Hensley ML, Alektiar KM, Gomez J, Gardner GJ, Chi DS, Barakat RR and Abu-Rustum NR: Patterns of recurrence in 1988 FIGO stage IC endometrioid endometrial cancer. Gynecol Oncol 125: 99-102, 2012.

18. Mariani A, Webb MJ, Keeney GL, Lesnick TG and Podratz KC: Surgical stage I endometrial cancer: Predictors of distant failure and death. Gynecol Oncol 87: 274-280, 2002.

19. Lindauer J, Fowler JM, Manolitsas TP, Copeland LJ, Eaton LA, Ramirez NC and Cohn DE: Is there a prognostic difference between depth of myometrial invasion and the tumor-free distance from the uterine serosa in endometrial cancer? Gynecol Oncol 91: 547-551, 2003.

20. Chattopadhyay S, Cross P, Nayar A, Galaal K and Naik R: Tumor size: A better independent predictor of distant failure and death than depth of myometrial invasion in international federation of gynecology and obstetrics stage I endometrioid endometrial cancer. Int J Gynecol Cancer 23: 690-697, 2013.
21. Zaino RJ, Kurman RJ, Diana KL and Morrow CP: Pathologic models to predict outcome for women with endometrial adenocarcinoma: The importance of the distinction between surgical stage and clinical stage-a gynecologic oncology group study. Cancer 77: 1115-1121, 1996.

22. Larson DM, Connor GP, Broste SK, Krawisz BR and Johnson KK: Prognostic significance of gross myometrial invasion with endometrial cancer. Obstet Gynecol 88: 394-398, 1996.

23. Patel S, Portelance L, Gilbert L, Tan L, Stanimir G, Duclos M and Souhami L: Analysis of prognostic factors and patterns of recurrence in patients with pathologic stage III endometrial cancer. Int J Radiat Oncol Biol Phys 68: 1438-1445, 2007.

24. Mariani A, Webb MJ, Keeney GL, Calori G and Podratz KC: Hematogenous dissemination in corpus cancer. Gynecol Oncol 80: 233-238, 2001.

25. Gemer O, Uriev L, Harkovsky T, Peled R, Ben-Dor D, Barak F and Seqal S: The significance of the degree of myometrial invasion in patients with stage IB endometrial cancer. Eur J Gynaecol Oncol 25: 336-338, 2004

26. Alektiar KM, McKee A, Lin O, Venkatraman E, Zelefsky MJ, Mychalczak BR, Mckee B, Hoskins WJ and Barakat RR: The significance of the amount of myometrial invasion in patients with Stage IB endometrial carcinoma. Cancer 95: 316-321, 2002.

27. Baloglu A, Bezircioglu I, Cetinkaya B and Hicyilmaz L: Prospective clinical study of the association between plasma level of free IGF-1 and myometrial invasion min patients with endometrial adenocarcinoma. Ginekol Pol 81: 501-505, 2010.

28. Schneider J, Martinez-Arribas F and Torrejón R: Nup88 expression is associated with myometrial invasion in endometrial carcinoma. Int J Gynecol Cancer 20: 804-808, 2010

29. Takama F, Kanuma T, Wang D, Kagami I and Mizunuma H: Oestrogen receptor beta expression and depth of myometrial invasion in human endometrial cancer. Br J Cancer 84: 545-549. 2001.

30. Soeda S, Nakamura N, Ozeki T, Nishiyama H, Hojo H, Yamada H, Abe M and Sato A: Tumor-associated macrophages correlate with vascular space invasion and myometrial invasion in endometrial carcinoma. Gynecol Oncol 109: 122-128, 2008.

31. Sakai K, Yamagami W, Susumu N, Nomura H, Kataoka F, Banno K, Tsuda H and Aoki D: Pathological factors associated with omental metastases in endometrial cancer. Eur J Gynaecol Oncol 36: 397-401, 2015.

32. Turan T, Hizli D, Sarici S, Boran N, Gundogdu B, Karadag B, Tulunay G and Kose MF: Is it possible to predict para-aortic lymph node metastasis in endometrial cancer? Eur J Obstet Gynecol Reprod Biol 158: 274-279, 2011.

33. Hétu V, Petignat P, Wu Y, Drouin P, Sauthier P, Provencher D and Gauthier P: Positive adnexal or uterine serosal involvement in stage IIIC endometrial cancer is an adverse factor for recurrence. Gynecol Obstet Invest 67: 173-177, 2009.

34. Jobsen JJ, Naudin Ten Cate L, Lybeert ML, Scholten A, van der Steen-Banasik EM, van der Palen J, Stenfert Kroese MC, Slot A, Schutter EM and Siesling S: Outcome of Endometrial Cancer Stage IIIA with Adnexa or Serosal Involvement Only. Obstet Gynecol Int 2011: 962518, 2011.

35. Guntupalli SR, Zighelboim I, Kizer NT, Zhang Q, Powell MA, Thaker PH, Goodfellow PJ and Mutch DG: Lymphovascular space invasion is an independent risk factor for nodal disease and poor outcomes in endometrioid endometrial cancer. Gynecol Oncol 124: 31-35, 2012

36. Maggi R, Lissoni A, Spina F, et al: Adjuvant chemotherapy vs radiotherapy in high-risk endometrial carcinoma: results of a randomised trial. British journal of cancer 95: 266-271, 2006 